Forum 2017 · 32:108

DOI 10.1007/s12312-017-0230-1

Online publiziert: 22. Februar 2017

๑) Springer Medizin Verlag GmbH 2017

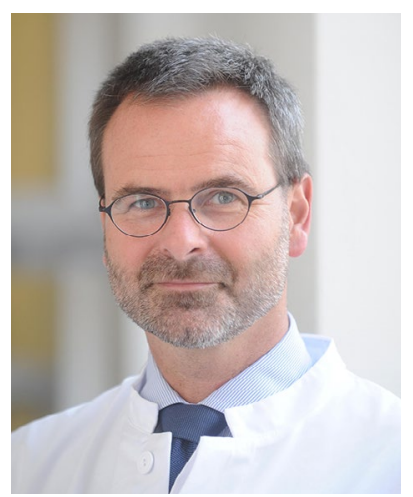

Schwerwiegende Erkrankungen wie Krebs sind nicht nur im medizinischen Sinn systemische Erkrankungen, sie wirken sich auch auf das soziale Gefüge der Betroffenen aus - auf Partnerschaft, Familien- und Berufsleben. Gerade beim Thema „Krebs und Partnerschaft" fühlen sich Patienten oft alleine gelassen. Das vorliegende Heft greift medizinische und psychosoziale Aspekte aus diesem Bereich auf - ein, wie ich finde, wichtiges Thema.

Aus gegebenem Anlass möchte ich an dieser Stelle noch auf ein anderes, wichtiges Thema eingehen. Mit Spannung war die Entscheidung des Innovationsausschusses über die Projekte erwartet worden, die für die nächsten drei Jahre durch den Innovationsfonds gefördert werden. Seit Januar 2017 ist es offiziell: Im Rahmen des Innovationsfonds ist unter anderem der Aufbau eines neuen Krebsregisters zur besseren Bewertung von Nutzen und Schaden innovativer Krebsmedikamente geplant. Zu diesem Zweck soll das

\title{
Peter Albers
}

Präsident der Deutschen Krebsgesellschaft e.V.

\section{Doppelte Register - doppelter Aufwand?}

geplante Register personenbezogene Daten über den Einsatz neuer Arzneimittel beim metastasierten Brust- und Prostatakrebs sowie dem multiplen Myelom sammeln und auswerten.

Das Projektziel ist wichtig und verdient jede erdenkliche gemeinsame Anstrengung: Seit langem schon mahnen Fachexperten an, dass die Datengrundlagen aus Zulassungsstudien nicht ausreichen, und fordern zusätzliche Versorgungsdaten zur Beurteilung des wirklichen Nutzens eines neu zugelassenen Medikaments. Ja, wir brauchen solche Daten. Dennoch sei die Frage erlaubt, ob wir dafür wirklich ein komplett neues Register benötigen. Denn mit den klinischen Krebsregistern steht bereits ein Instrument zur Verfügung, das den gesamten Krankheitsverlauf eines Krebspatienten, von der Erstdiagnose bis zum Rückfall, lückenlos dokumentieren kann. Liegt es da nicht nahe, beim Aufbau des neuen Registerprojekts Synergien mit den bestehenden klinischen Krebsregistern zu nutzen?

\section{》) Synergien mit den klinischen Krebsregistern sollten genutzt werden}

Der gesetzliche Rahmen für die bundesweite Erfassung personenbezogener Krankheitsdaten in klinischen Krebsregistern besteht bereits seit 2013 in Form des sogenannten Krebsfrüherkennungsund -registergesetzes (KFRG). Demnach sind alle Bundesländer in Deutschland aufgefordert, bis Ende 2017 klinische Krebsregister einzurichten, in einigen Bundesländern sind sie bereits aktiv. Bei der Ausgestaltung des gesetzlichen Rahmens für die klinischen Krebsregister haben sich alle Beteiligten ausdrücklich auf eine einheitliche, datensparsame Basisdokumentation und die Vermeidung von Doppelerfassungen durch die Leistungserbringer verständigt.

Ohne Kenntnisse der vorherigen Krankengeschichte wird die Interpretation der Daten aus dem neuen Register schwierig. Und viele der Anforderungen an die klinischen Krebsregister werden auch für das neue Register gelten. $\mathrm{Ob}$ und wie eine $\mathrm{Zu}$ sammenarbeit zwischen beiden Registern stattfinden soll und wie die Schnittstelle aussehen könnte, war bei der Förderentscheidung aber offenbar kein Kriterium. Nutzt man die Synergiemöglichkeiten mit den klinischen Krebsregistern nicht, dann kostet die Entwicklung des neuen Registers zusätzliches Geld und der Aufwand wird größer. Ich vertraue auf eine konstruktive Einstellung aller Beteiligten.

Ihr

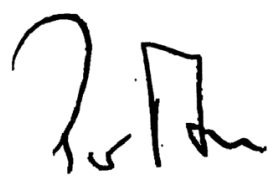

Ihr Peter Albers

Präsident der Deutschen Krebsgesellschaft 